BABELAO 3 (2014), p. 109-124

(C) ABELAO (Belgium)

\title{
À propos de l'Évangile selon Saint Jean de la British and Foreign Bible Society en berbère tachelhit (Maroc)
}

Par

\section{Naïma Afif}

Université catholique de Louvain

L

'Evangile de Jean en berbère tachelhit ${ }^{1}$ dont il est question dans le présent article a été publié au début du $20^{\mathrm{e}}$ siècle sous les auspices de la British and Foreign Bible Society ${ }^{2}$ par des missionnaires protestants de la Southern Morocco Mission ${ }^{3}$. Cette traduction biblique, relativement tardive, répondait au défi de

${ }^{1}$ Le berbère appartient à la famille des langues afro-asiatiques qui comprend les langues tchadiennes, l'égyptien, le couchitique et le lybico-berbère. Le tachelhit est le berbère parlé au Sud-Ouest du Maroc, à côté du rifain (au Nord) et du tamazight (au centre).

${ }^{2}$ Société fondée à Londres en 1804 dans le but de promouvoir la diffusion de la Bible en langues étrangères, cf. L.J. BALL, "British and Foreign Bible Society ", dans H.J. HiLlerBRAND (éd.), The Encyclopedia of Protestantism, vol. 1, Routledge, New York-Londres, 2004, p. 304-306.

3 St. John Gospel, British and Foreign Bible Society, Alger, 1906, cf. T.H. DARLOW et H.F. MOULE, Historical Catalogue of the Printed Editions of Holy Scripture in the Library of the British and Foreign Bible Society. Reprinted with the Permission of 
transmettre le message évangélique en pays musulman dans une culture essentiellement orale. Afin de mettre le texte à portée des locuteurs chleuhs, les traducteurs ont ainsi procédé à de nécessaires adaptations du quatrième évangile à la réalité berbère (institutions, vocabulaire du culte, nomenclature...) et formulé pour la première fois les notions du vocabulaire chrétien en tachelhit.

Ecrite en alphabet arabe ${ }^{4}$, cette version de l'Evangile de Jean a retenu l'attention des berbérisants qui en ont produit des transcriptions à base latine (A. Roux, H. Stroomer) ${ }^{5}$ mais n'a, à ce jour, pas fait l'objet d'une étude particulière. En outre, bien que l'activité missionnaire chrétienne au Maroc durant les périodes précoloniale et coloniale ait suscité des recherches ${ }^{6}$, les textes bibliques traduits en berbère dans ce contexte, constituent, eux, un champ peu exploré ${ }^{7}$. On s'efforcera ici d'apporter un premier éclairage sur la question en guise de contribution historique ${ }^{8}$ et philologique.

\section{Cadre missionnaire de la traduction et inventaire des éditions}

Lorsque la Southern Morocco Mission est fondée à Glasgow en 1888, elle se donne pour objectif de combler l'absence de mouvement d'évangélisation dans le Sud du Maroc auprès des populations berbères, arabes et juives? ${ }^{9}$ La mission s'implante

The British and Foreign Bible Society, London, vol. 2/3 (Polyglots and Languages other than English), Kraus Reprint Corporation, New York, 1963, p. 1364.

${ }^{4}$ Celui-ci est utilisé pour la notation du berbère depuis le Haut Moyen-Age, cf. N. VAN DEN BOOGERT, "Medieval berber orthography », dans S. CHAKER \& A. ZABORSKI (éds.), Etudes Berbères et Chamito-Sémitiques. Mélanges offerts à KarlG. Prasse, Peeters, Paris-Louvain, 2000, p. 357-377. Bien que la culture berbère relève principalement de l'oralité, une tradition écrite à caractère religieux est attestée en tachelhit à partir du $17^{\mathrm{e}}$ siècle. Le choix des traducteurs correspond donc à un usage sinon répandu, du moins consacré dans le Sud marocain. Signalons qu'actuellement au Maroc, un alphabet berbère, le néo-tifinagh, est en usage dans le cadre de la standardisation du berbère, la langue amazigh.

${ }^{5}$ Cf. chapitre 1.

${ }^{6}$ Pour une vision générale de la présence chrétienne au Maroc à partir de la seconde moitié du $19^{\mathrm{e}}$ siècle, voir J. BAÏDA et V. FEROLDI, «Une mémoire commune en devenir : chrétiens et musulmans au Maroc (1856-1985)» dans V. FEROLDI (dir.), Chrétiens et musulmans en dialogue, les identités en devenir : travaux $d u$ Gric (1996-2003), L'Harmattan, Paris, 2003, p. 53-83. Pour l'histoire des missions protestantes au Maroc, voir J.-L. MIÈGE, «Les missions protestantes au Maroc (1875-1905)", dans Hespéris: Archives berbères et bulletin de l'Institut des Hautes Etudes Marocaines, 42, 1955, p. 153-192.

${ }^{7}$ Citons néanmoins le Pater Noster traduit en tachelhit par J. Jones et publié dans l'Oratio Dominica de Chamberlayne au $18^{\text {e }}$ siècle. H. Stroomer en a livré une analyse détaillée, cf. H. STROOMER, "An Early European Source on Berber. Chamberlayne (1715) » dans S. ChAKER \& A. ZABOrSKI (éds.), ibid., p. 303-316.

${ }^{8}$ Les informations que l'on fournira sont en partie tributaires des comptes rendus de la mission qu'il a été possible de consulter.

9 Jusqu'alors, les missions protestantes dans la région s'étaient surtout intéressées à la minorité israélite locale. Une première tentative d'évangélisation avait été menée à Mogador (l'actuelle Essaouira) dès 1834, mais elle s'avéra infructueuse, J.-L. MIĖGE, ibid., p. 154-155. 
d'abord à Mogador en 1889 sous la direction du médecin et missionnaire presbytérien Cuthbert Nairn, bientôt rejoint par d'autres agents écossais. Une année plus tard, le siège central est établi à Marrakech et l'œuvre de prédication de la mission, doublée d'une importante activité sociale (soins médicaux, création d'écoles, etc.), s'étend bientôt à Mazagan (l'actuelle el-Jadida), Safi et Amizmiz (à l'intérieur des terres). Afin de toucher un public large, C. Nairn et D. Muir ${ }^{10}$ se familiarisent avec les langues vernaculaires (l'arabe et le berbère) et en 1904, avec l'aide d'un soussi de Taroudant ${ }^{11}$, ils achèvent la traduction de l'Evangile selon Saint Jean en tachelhit qui sera publiée en 1906 par la British and Foreign Bible Society ${ }^{12}$. Cette édition composée de 166 pages écrites en caractères arabes de style maghribi ${ }^{13}$ (cf. spécimen, p. 20) est la reproduction lithographiée d'une copie préparée par un agent de la Société biblique à Alger du nom de $\mathrm{J}$. May ${ }^{14}$. Publiée sans nom

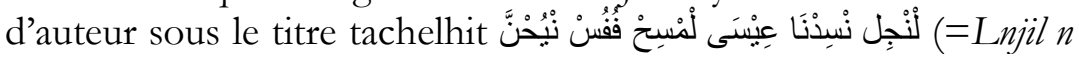
Sidnâ Eîsâ lmsih f ufus n Yuhnna), elle est accompagnée, au bas de la dernière page, d'une brève description en français : Evangile selon Saint-Jean traduit en Chelh' ${ }^{15}$, dialecte Berbère du Sous Marocain. Le texte est diffusé grâce au travail de colportage, tandis que dans les dispensaires et hôpitaux de la mission, la lecture de passages précède les consultations, recevant souvent un accueil mitigé ${ }^{16}$. La traduction fait ensuite l'objet d'une révision par les soins des traducteurs en $1925^{17}$ et sera plusieurs fois imprimée ${ }^{18}$.

A l'issue de quarante années de prédication, il est difficile de déterminer le nombre ou même l'éventualité de convertis au christianisme dans le Sud du territoire marocain. C. Nairn dresse

${ }^{10} \mathrm{D}$. Muir était chargé de l'évangélisation des populations juives, cf. « Work among Jews ", dans Southern Morocco Mission. Founded 1888. A Review of the Work, Pickering \& Inglis, Glasgow, 1918, p. 9.

11 J.-L. MiĖGE, ibid., p. 169. Un « soussi » est un habitant du Souss, région berbérophone (tachelhit) située au Sud-Ouest du Maroc.

12 « The Gospel of St John in Susiya, translated by Cuthbert Nairn and David Muir of the South Morocco Mission, was almost ready for publication in 1904 », cf. W. CAnton, A bistory of the British and Foreign Bible Society, J. Murray, Londres, vol. 5, 1910, p. 11.

${ }^{13}$ L'écriture de style maghribi présente les particularités diacritiques suivantes : absence de point(s) sur le nun final et le qaf, point suscrit sur le fa. Des aménagements graphiques sont apportés aux lettres arabes pour combler l'absence de correspondance phonétique avec le tachelhit (par exemple le phonème /g/, noté au moyen de la lettre kaf et de trois points souscrits ou suscrits).

14 Cf. T.H. DARLOW et H.F. Moule, op. cit. Seul un titre anglais est mentionné : St.John Gospel.

15 De l'arabe شلحة, « tachelhit ».

${ }^{16}$ J.-L. MiÈGE, ibid., p. 174. L'évolution du contexte politique marocain (le Maroc est sous protectorat français à partir de 1912), accentuera le climat de défiance de la population vis-à-vis de la présence européenne quelle qu'elle soit.

17 G.E. Coldham, A Bibliography of Scriptures in African Languages, vol. 2, British and Foreign Bible Society, Londres, 1966, p. 585.

18 Pour les rééditions et/ou réimpressions de la traduction, on consultera éventuellement ID., Supplement (1964-1974) to A Bibliography of Scriptures in African Languages, British and Foreign Bible Society, Londres, 1975. 
cependant un bilan optimiste de l'œuvre d'évangélisation ${ }^{19}$ et de l'extension de la mission, les principales villes et villages en-deçà d'Agadir étant désormais occupés ${ }^{20}$. Après la mort de son surintendant (en 1944), la Southern Morocco Mission poursuivra ses activités et fusionnera, en 1959, avec la North Africa Mission ${ }^{21}$.

Depuis, d'autres textes bibliques ont été traduits en tachelhit par l'United Bible Societies: l'Evangile de Marc (1994), les Epîtres de Pierre (1994) et l'intégralité du Nouveau Testament (1998).

En 2000 (soit un peu moins d'un siècle après sa parution), la traduction de C. Nairn et D. Muir a néanmoins connu un regain d'intérêt tant sur le plan confessionnel que scientifique, avec une transcription à base gréco-latine publiée par l'Association chrétienne d'expression berbère, en collaboration avec le Département des langues et cultures du Moyen-Orient islamique de l'université de Leyde ${ }^{22}$. Cette édition a été réalisée par H. Stroomer selon un système morpho-phonologique, conformément à l'un des usages répandus dans les études berbères ${ }^{23}$. Le texte de la traduction est de facture simple, ce qui - en plus de l'importance doctrinale du quatrième évangile - explique très certainement sa réactualisation auprès des berbères chrétiens.

19 " It is impossible to say how many Moslems and Jews who have been in frequent and close contact with the missionaries may have believed unto salvation. One cannot doubt that numbers during the course of the years have believed the message of the Gospel, although they made no pronounced profession of faith ", cf. "Gospel Preaching », dans Southern Morocco Mission. Founded 1888. A Review of the Work, Pickering \& Inglis, Glasgow, 1926, p. 4.

20 "Now every town of any importance is occupied, except Agadir and Tarudant, which are still barred to us ", cf. "These Fourty Years (1888-1928) ", dans Southern Morocco Mission. Founded 1888. A Review of the Work, Pickering \& Inglis, Glasgow-Londres, 1928, p. 5.

21 R. BENEDETTO et D.K. MCKIM, Historical Dictionary of the Reformed Churches, The Scarcrow Press (Historical Dictionaries of Religions, Philosophies, and Movements, 99), Lanham-Toronto-Plymouth, 2010, $2^{\text {de }}$ édition, p. 337.

${ }^{22}$ Je remercie le Prof. H. Stroomer de m'en avoir aimablement fait parvenir une copie, H. STROOMER, L'Evangile selon Saint Jean en berbère Tachelhiyt (Maroc), Paris-Leyde, 2000. Ce texte a été établi à partir de St. John's Gospel in Shilha : Susi dialect, British and Foreign Bible Society, 1937. Il présente des variantes lexicales par rapport à la première édition de la traduction (1906), peut-être parce qu'il s'agit d'une réimpression de la révision de 1925. Sans nous prononcer sur le sujet, et pour donner une illustration de certains de ces écarts, les variantes de Stroomer qui apparaissent dans les exemples présentés dans cet article, basé sur l'édition princeps, seront signalées et commentées en note.

${ }^{23}$ C'est celui qui a été adopté dans la présente publication. Signalons qu'il existe aussi un exemplaire de la traduction de l'Evangile de Jean en tachelhit dans le fonds de manuscrits arabes et berbères d'Arsène Roux : le ms 138. Composée de 106 pages, cette copie en caractères latins diacrités a été faite dans un simple cahier par l'un des étudiants du fameux berbérisant, cf. N. VAN DEN BoOgert, Catalogue des manuscrits arabes et berbères du Fonds Roux (Aix en Provence), Travaux et documents de l'Iremam, 18, Aix-en-Provence, 1995, p. 77. 


\section{Phonologie du texte ${ }^{24}$ et système de transcription}

Voici - pour se borner à l'essentiel - les principales caractéristiques du système phonologique du berbère tachelhit ${ }^{25}$ :

- le vocalisme est ternaire : $a / i / u$ (sans opposition brève/longue) ;

- comme dans les autres parlers berbères, le système consonantique est caractérisé par la tension articulatoire (que l'on indiquera par le redoublement de la lettre) et l'emphase (notée par un point suscrit). Toutefois, on constate plus particulièrement:

a) la réalisation occlusive des consonnes $/ \mathrm{b} /, / \mathrm{d} /, \mathrm{t} /$, $/ \mathrm{g} /, / \mathrm{k} /$;

b) une tendance à la labio-vélarisation des consonnes vélopalatales (indiquée par ");

c) l'absence de shewa, insensible à la prononciation (elle n'est dès lors par transcrite).

Le texte de l'édition en alphabet arabe présente quelques libertés par rapport à ce système phonologique de base : ni la labio-vélarisation ni l'emphase de la sonore /z/ ne sont restituées à l'écrit, mais le texte vocalisé offre un support de base suffisant pour le lecteur chleuh, sans doute invité à restituer de lui-même les articulations manquantes.

En dépit de son homogénéité, le tachelhit présente des variations phonétiques régionales comme par exemple l'aspiration du $k$ en $k^{b}$ (ex : «toi » au féminin = kmmi (Ifrane, Anti-Atlas) et $k^{b} m m i$ (Argana, province de Taroudant). De ce point de vue, le texte de la traduction ne présente pas de particularités significatives. Signalons toutefois quelques phénomènes isolés dont : a) la réalisation de $/ \mathrm{j} /$ en $/ \mathrm{dj} /$ dans adj itt « laisse-la » (Jn 12,7) et tadjim t a iddu « ... et laissez-le s'en aller » (Jn 11,44), mais ajjat « laissez » (Jn 18,8) ; b) l'assimilation à distance de /s/ en /š/ dans iškšsm «il fit entrer » en Jn 18,16 (< iskšmm).

\section{Eléments d'adaptation de l'Evangile de Jean en ta- chelhit}

Sans prétendre à l'exhaustivité, deux aspects seront envisagés dans la présente analyse :

1) les spécificités du vocabulaire religieux de la traduction que l'on abordera succinctement ;

2) les éléments d'adaptation culturelle ayant une influence exégétique (ou interprétative) qui seront plus longuement développés.

${ }^{24}$ Cette analyse est établie à partir de l'édition de 1906.

25 S. CHAKER, «Chleuh», dans Encyclopédie berbère, 13, Edisud, Aix-enProvence, 1994, p. 1926-1933. Voir aussi M.G. Kossmann et H.J. STROOMER, "Berber Phonology ", dans A.S. KaYe (éd.), Phonologies of Asia and Africa, vol. 1, Eisenbrauns, Winona Lake, 1997, p. 467-468. 


\section{1. Lexique de traduction : caractéristiques générales}

Sur le plan lexical, de nombreux mots arabes ont été introduits en berbère. Ce phénomène étant courant dans le vocabulaire religieux ${ }^{26}$, son importance dans la traduction de l'Evangile de Jean en tachelhit ne constitue pas une exception ${ }^{27}$. Ces emprunts lexicaux sont de plusieurs types (mots berbérisés ou translittérés, avec parfois des glissements sémantiques). Ils ont subi dans la plupart des cas des modifications phonologiques mineures, notamment par réduction du système vocalique et peuvent être empruntés avec l'article arabe: Injil < الانجيل

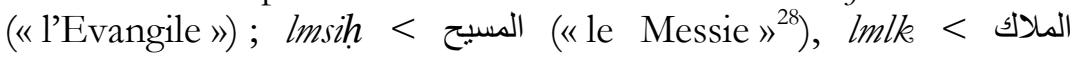
(«l'ange »).

- Les traducteurs ayant employé le lexique arabomusulman intégré en berbère tachelhit, le vocabulaire biblique de la traduction ne présente pas de correspondances lexicales avec l'arabe chrétien, excepté lorsque le concept exprimé dans l'Evangile est absent de la tradition musulmane (c'est le cas du terme "baptiser", cf. Jn 3,26). La formulation du vocabulaire biblique est donc principalement calquée par défaut sur la terminologie islamique :

d'Adam »)

- 《le Fils de l'Homme» = Yus n bnadm > بنو آدم (fils

- «le Fils de Dieu» = Yus n Rbbi<ب («Seigneur», suivi du suffixe possessif de la $1^{\text {ère }}$ personne du singulier ${ }^{30}$ )

- «l'Agneau de Dieu»= Alqqay $n$ Rbbi

- 《le roi d'Israël »= agllid¹ n Bani32 Israyl > بنو إسرائيل ("enfants d'Israël ») en Jn 1,49.

${ }^{26} \mathrm{~N}$. van den Boogert et M. Kossmann ont mis en évidence l'ancienneté du phénomène d'emprunt arabe dans le cadre de la terminologie religieuse islamique. Il présente dans les cas mentionnés (prière rituelle, jeune, mosquée...) une relative homogénéité dans l'ensemble des parlers berbères, $\mathrm{cf}$. $\mathrm{N}$. VAN DEN BOOGERT et M. KOSSMANN, "Les premiers emprunts arabes en berbère ", dans Arabica, 44, fasc. 2, avr. 1997, p. 317-322.

${ }^{27}$ Dans la traduction, ces emprunts portent également sur le lexique usuel. Il est cependant difficile de déterminer s'il s'agit d'occurrences occasionnelles (dues par exemple au contexte urbain des traducteurs, Marrakech) ou d'emprunts véritablement lexicalisés.

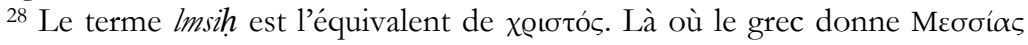
(translittération du mot hébreu et araméen משיח), le texte tachelhit emploie à son tour la translittération du mot grec : Masiyya.

${ }^{29}$ Le mot yus de la racine berbère $w$, cf. V. BLAžEC, «Toward the berber kinship terminology in the afroasiatic context», dans K. NAÏT-ZERRAD (éd.), Articles de linguistique berbère. Mémorial Werner Vycichl, L’Harmattan (Tira Langues, littératures et civilisation berbères), Paris, 2002, p. 117.

${ }^{30}$ Intégré dans le lexique panberbère. Sur les autres noms de Dieu en berbère, voir J. LANFRY, « Dieu » dans Encyclopédie Berbère, 15 (Daphnitae-Djado), Aix-en-Provence, Edisud, 1995, p. 2313-2321. Dans la traduction, Rbbi est aussi utilisé pour кú@ıos (lorsqu'il s'agit de Dieu), comme c'est le cas dans la

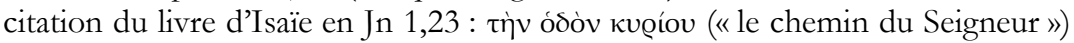
$=$ a jarass $n$ Rbbi. Toutefois, lorsque Jésus rencontre la Samaritaine près du puits de Jacob, elle l'appelle Sidi (pour Kúpıe), formule de respect, « Seigneur ».

${ }^{31}$ Le terme agllid (agellid, ajellid, ayellid « roi, monarque, prince, souverain » est issu de la racine gld, attestée dans l'ensemble du domaine berbère et présente sur des inscriptions libyques, cf. K. NAÏT-ZERRAD, Dictionnaire des racines 
- L'onomastique des figures communes à la tradition musulmane et au christianisme est islamisée : ainsi, Jésus est $\sum \hat{\imath} \hat{a}$ ou Sidnâ Eîsâ et Jean-Baptiste, Yahya $\hat{a}^{33}$, mais le nom de l'évangéliste, lui, correspond à la forme arabe chrétienne (Yuhanna). Les anthroponymes d'origine grecque, hébraïque et latine sont également arabisés : Andrawus (André), Simean Butrus (Simon Pierre), Yuna (Jonas), Bilatus (Pilate) ou encore Yahuda Lsxariyuti (Judas Iscariote).

En somme, on peut dire qu'aucune rupture n'est opérée dans la conversion du message évangélique en tachelhit. Le substrat religieux du milieu de réception est simplement enrichi de nouveaux éléments doctrinaux.

\section{2. Réduction de la distance culturelle : exemples} significatifs

Afin de rendre le texte intelligible auprès des lecteurs/locuteurs chleuhs, des rapprochements entre le cadre culturel de l'évangile johannique et le milieu berbère sud marocain ont nécessairement été établis. Pour signifier " prétoire ${ }^{34}$ ou «César », les traducteurs ont en effet été amenés à trouver des équivalences dans la culture d'arrivée (=tigmi $n \operatorname{lm} x z^{35}{ }^{35}$, agllid $n \mathrm{Rrum}^{36}$ ), sous peine de livrer un texte obscur à leur auditoire.

Sans dresser une liste complète de correspondances, on examinera dans la section qui suit l'utilisation des termes $\mathrm{P} \alpha \beta \beta$,

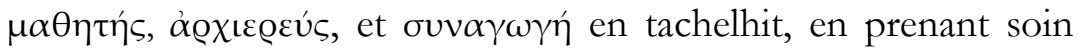
d'en évaluer les rapprochements avec le cadre berbère. Les exemples retenus pour l'analyse proprement dite seront cités dans le contexte immédiat des versets où ils apparaissent ${ }^{37}$. En raison des principes de transcription adoptés ${ }^{38}$, le texte tachelhit présente un écart par rapport à l'original en alphabet arabe (= édition princeps, 1906), aussi, dans un souci de précision, la translittération du texte d'origine sera donnée en bas de page.

berbères (formes attestées) : D-Gey, vol. 3, Peeters (M. S. Ussun amazi $\gamma, 19)$, ParisLouvain, 2002, p. 773-774. Voir aussi S. CHAKER, «Terminologie libyque des titres et des fonctions ", dans Linguistique berbère : études de syntaxe et de diachronie, Peeters, Paris-Louvain, 1995, p. 173.

32 Bani] Banu, Stroomer.

${ }_{33}$ Notons le maintien des voyelles longues dans ces emprunts de l'arabe.

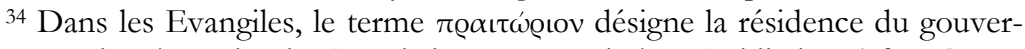
neur romain où ce dernier jugeait les cas soumis à sa juridiction (cf. F.G. VIGOuroux, Dictionnaire de la Bible, vol. 5/1, 1912, col. 621- 639).

35 Littéralement : «la maison du gouvernement», traduction de l'arabe

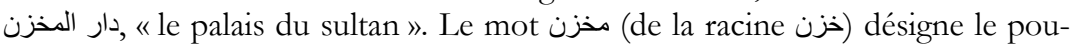
voir politique en place.

36 De l'arabe classique روم, «Romains 》 (de l'Empire d'Orient).

37 A titre indicatif, certains passages divergents du texte tachelhit seront mis en parallèle avec le texte grec (= E. NeSTLE et K. ALAND, Novum Testamentum Graece, Deutsche Bibelgesellschaft, Stuttgart, 28e édition, 2012).

${ }^{38} \mathrm{La}$ forme de base des cas d'assimilation est restituée ; les éléments grammaticaux, segmentés arbitrairement dans le texte en caractères arabes, sont séparés de façon à mettre en évidence les composants syntaxiques des énoncés. 
On a cru utile d'accompagner les exemples d'indications lexicographiques ainsi que d'éléments de grammaire comparée, susceptibles de profiter aux sémitisants. Enfin, une traduction permet de se familiariser avec le texte tachelhit.

\section{- Rabbi}

Le mot «Rabbi » est expliqué dans le premier chapitre du texte johannique $(\mathrm{Jn} 1,38)^{39}$. En tachelhit, il est traduit par la particule vocative $a(=\hat{0})$ suivie du mot lfqih, de l'arabe فق vant et versé dans la jurisprudence musulmane ${ }^{40}$. En arabe classique, le فقيه (faqīh) est «le jurisconsulte » ou le savant en droit islamique ${ }^{41}$, mais dans le domaine berbère, de même qu'en arabe dialectal marocain, le terme peut désigner le maitre d'école coranique $^{42}$ et/ou le «lettré ${ }^{43}$. En tachelhit du Souss, E. Destaing lui donne le sens « d'instituteur " ${ }^{44}$ et " professeur » en soulignant que l'enseignement élémentaire est dispensé par le talb (de l'arabe طلب), tandis que " pour un enseignement plus élevé, on s'adresse à l'Ealim ${ }^{45}$ (de l'arabe (علم) cet enseignement ayant, il s'entend, un caractère religieux :

\section{$\cdot$ Jn 1,38 :}

Igriwl $\sum \hat{\imath} \hat{s} a \hat{a}$ ar tn ittmnad tfurn t, inna yasn : Mat tsiggilm? Nnan as : Rbbi mani at tilit? (tga Imena $n$ Rbbi, a lfqib) = (Jésus se retourna, et

\footnotetext{
${ }^{39}$ Le terme P $\alpha \beta \beta \hat{~}$ (« Maître »), d’origine araméenne (רב suivi du suffixe de la $1^{\text {ère }}$ personne du singulier, "mon Maître ») est translittéré tel quel dans le texte grec.

40 A.B. KAZIMIRSKI, Dictionnaire Arabe-Français contenant toutes les racines de la langue arabe, leur dérivés, tant dans l'idiome vulgaire que dans l'idiome littéral, ainsi que les dialectes d'Alger et de Maroc, vol. 2, Maisonneuve, Paris, 1860, p. 623.

${ }^{41}$ Lane lui donne un sens plus large : « any one possessing knowledge of a thing ». E.W. LANE, An Arabic-English Lexicon, vol. 1, Librairie du Liban, Beyrouth, 1968, p. 2429.

${ }^{42}$ C'est le cas dans de nombreux dialectes, cf. D.B. MACDONALD, « Faḳīh » dans The Encyclopaedia of Islam, vol. 2., nouvelle édition, Brill, Leiden, 1991, p. 756. Pour l'arabe marocain, voir W. MARÇAIS, Textes arabes de Tanger. Transcription, traduction annotée, glossaire, vol. 4, Paris, 1911, p. 415. Dans son lexique, de Prémare lui donne le sens de «juriste » et précise que le mot peut désigner "l'imam de la prière du vendredi ». Le même terme avec chute du • (فقي) désigne le «lettré », le «maître d'école coranique ». Plus particulièrement, a lfqih, c'est-à-dire " ô maitre » servirait aussi d'interjection pour interpeller un cadi, le gouverneur d'un village ou d'une ville, cf. A.-L. DE PRÉMARE, Dictionnaire Arabe-Français. Langue et culture marocaines, vol. 10, Editions L'Harmattan, 1998, p. 138.

43 J. Houtsonen, "Traditional Quranic Education in a Southern Moroccan Village ", dans International Journal of Middle East Studies, vol. 26/3, Aug. 1994, p. 489-490.

${ }^{44}$ E. Destaing, Vocabulaire Français-Berbère. Etude sur le tachelhît du Soûs, Paris, 1938 , p. 158.

45 Ibid., p. 232.
} 
voyant qu'ils le suivaient, leur dit: Que voulez-vous ? Ils lui dirent : Rabbi où demeures-tu ? («Rabbi » signifie ${ }^{46}$ « ô maittre »).

Dans l'interrogatif de lieu manì (« où ») on retrouve le thème en $m$ exprimant l'interrogation commun au groupe sémitique et afro-asiatique ${ }^{47}$ et la préposition $\gamma$ (《dans »). Le mot lmena, de l'arabe ("signification»), précédé de l'article, est intégré tel quel en tachelhit.

\section{$\cdot$ Jn 3,26 :}

... A lfqih, rwa lli stt inn didk ikekan $\gamma$ ugmmad ann $n$ Lurdunn ${ }^{48}$, lli $f$ tfkit tugga, ha ti ar ittemmad htta nttan... (=... O maitre, celui qui était avec toi sur l'autre rive ${ }^{49}$ du Jourdain (et) à qui tu as rendu témoignage, voici qu'il baptise lui aussi...)

Pour signifier «témoigner, rendre témoignage, être témoin » deux expressions berbères sont employées dans le texte tachelhit : kf tugga (littéralement « donner témoignage ») et $g$ inigi (" être témoin ») ${ }^{50}$. La racine $s h d$ est utilisée exceptionnellement en Jn 1,34 et correspond au sens de « rendre témoignage de quelque chose » mais aussi « prononcer les paroles sacramentelles constituant l'article de foi musulmane $»^{51}$. Ce verbe convient donc par analogie à la profession de foi de Jean qui, dans le passage concerné, témoigne de la divinité du Christ. Le verbe «baptiser» est rendu par la racine عui, en arabe chrétien, signifie «baptiser, administrer le sacrement du baptême à quelqu'un » ${ }^{52}$ à la $2^{\text {ème }}$ forme. En arabe, le verbe se construit avec l'accusatif de personne mais dans le texte en tachelhit, il est plus généralement intransitif et se présente dans tous les cas à l'aoriste intensif.

\section{$\cdot$ Jn 3,10 :}

Iwajb Lîsâ, inna yas : Ha kiyyyi tgit lfqih n Bani Israyl, sul ur tssint limur $a d$ ? (= Jésus répondit (et) lui dit: Tu es maitre en Israël et tu ignores ces choses ? »)

Le pronom personnel indépendant $2 \mathrm{~m}$. s kiyyi est en partie formé à partir du suffixe personnel berbère de la $2 \mathrm{~m}$. s. $k$ (cf. $k(a)$ en akkadien et $k$ en égyptien $)^{53}$. La formule «en Israël» correspond à, littéralement: «des enfants

\footnotetext{
${ }^{46}$ Littéralement : « le sens de Rabbi est... ».

47 J.-C. HAELEWYCK, Grammaire comparée des langues sémitiques. Éléments de phonétique, de morphologie et de syntaxe, éditions Safran (Collection Langues et cultures anciennes, 7), Bruxelles, 2006, p. 110.

$48 n$ Lurdunn] Llurdunn. L'assimilation du $n$ (" de » introduisant le complément du nom) en $l$ est indiquée dans l'édition princeps. Le phénomène d'assimilation est ici rétabli dans sa forme syntaxique (idem chez Stroomer).

${ }^{49}$ Le mot $a g^{\prime \prime}$ mmad de la $\sqrt{ }$ panberbère $g m d$ « rive » suivi de ann (démonstratif d'éloignement) correspond à la « rive opposée à celle où l'on est», cf. K. NAÏT-ZERRAD, Dictionnaire des racines berbères (formes attestées): D-Gey, vol. 3, Peeters (M. S. Ussun amazi $\gamma$, 19), Paris/Louvain, 2002, p. 804.

${ }^{50}$ A propos de cet emploi, cf. P. GALANT-PERNET, «Traces de représentations archaïques dans le lexique et la morphologie berbères. Le cas de inigi ", dans K. NAÏT-ZERRAD (éd.), Articles de linguistique berbère..., p. 197-234.

51 A.B. KASIMIRSKI, ibid., vol. 1, p. 1279.

52 Ibid., vol. 2, p. 361

${ }^{53} \mathrm{Au}$ sujet de la formation des pronoms personnels affixes et indépendants en berbère, cf. S. CHAKER, «La parenté chamito-sémitique du berbère: un
} 
d'Israël ». Le terme limur (« choses») dérive de l'arabe أمر (même sens).

Le terme lfqib est employé pour traduire P $\alpha \beta \beta i$ (« Maittre »)

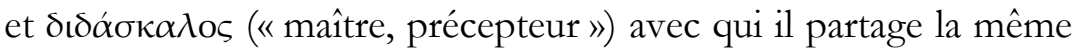
signification. Il présente donc (compte tenu de l'occurrence de ces deux termes dans l'Evangile johannique) la particularité de s'appliquer uniquement à Jésus, Jean-Baptiste et Nicodème.

\section{- Disciple}

Au terme «disciple », $\mu \alpha \theta \eta \tau \eta ́ s$, dont on compte plus de soixante-dix occurrences dans le quatrième évangile, correspond le mot amhdar (au pluriel imhdarn), de l'arabe حضر, "être présent », racine à partir de laquelle est dérivée la forme nominale en am- qui compose le nom d'agent en berbère ${ }^{54}$. En tachelhit, le mot amhdar désigne l'écolier qui suit généralement un enseignement coranique $^{55}$. Les imhdarn du texte johannique en tachelhit sont les disciples de Jean (1,35), les adeptes de Moïse (Jn 9,28) et bien entendu les disciples de Jésus :

\section{$\cdot$ Jn 4,31 :}

Walaynni lli ur ta d lkimn mddn ann, ar tthllaln imhdarn nns, ar as ttinin: Hak, at tšst, a lfqih. (= Mais comme ces personnes n'étaient pas encore arrivées, ses disciples l'entouraient d'attention (et) lui disaient : Tiens, mange, ô maitre).

«Mais comme ces personnes n'étaient pas encore arrivées » $=$ 'Ev $\tau \tilde{\omega}$

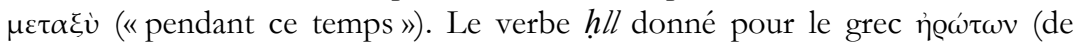
$\dot{\varepsilon} \varrho \omega \tau \alpha ́ \alpha$, «demander, interroger») est mentionné par Destaing sous l'entrée, « caresser $»^{56}$, «consoler $»^{57}$ et à la forme factitive : «bercer» (se dit d'un enfant $)^{58}$. L'usage de ce verbe vise certainement à souligner l'affection des disciples pour Jésus (d'où « l'entouraient d'attention »).

faisceau d'indices convergents", dans Linguistique berbère. Etudes de syntaxe et de diachronie, Paris-Louvain, Peeters, 1990, p. 233-235.

${ }^{54} \mathrm{Ce}$ préfixe est à rapprocher de $m u$ - en arabe et en akkadien, ma- en éthiopien, $m$ - en araméen et en hébreu, cf. J.-C. HAELEWYCK, ibid., p. 144.

55 Destaing lui donne simplement le sens d'écolier, en précisant que l'écolier plus âgé se dit Imsafer (de l'arabe سفر) ou encore le taleb. E. DESTAING, ibid, p. 102. Même s'il revêt d'abord un sens religieux, le mot amhlạ peut s'appliquer par extension à «toute personne en situation d'apprentissage » cf. A. BOUNFOUR, "Oralité et écriture: un rapport complexe », dans Revue de l'Occident musulman et de la Méditerranée, 44, 1987, p. 87.

${ }^{56}$ E. Destaing, ibid., p. 53.

${ }^{57}$ Ibid., p. 73.

58 Ibid., p. 37. 


\section{$\cdot$ Jn 1,35 :}

Asska yann day, ibidd Yahyâ nttan d sin à imhdarn nns. (= Le lendemain, Jean se tenait à nouveau en compagnie de deux ${ }^{59}$ de ses disciples).

\section{$\cdot$ Jn 9,28 :}

... Kiyyi a igan amhdar n ywann, imma nke" ni imhdarn n Musa an nga. (= C'est toi qui es son disciple ${ }^{60}$; nous, nous sommes les disciples de Moïse).

La relation des disciples avec leur maitre, lien de base de la communauté johannique, est calquée sur l'organisation de l'enseignement religieux en milieu islamo-berbère : utilisé conjointement avec lfqih pour "Rabbi », le terme amhdar compose un champ lexical cohérent pour le lecteur chleuh.

\section{- Grand prêtre}

L'autorité juive dans l'Evangile de Jean est représentée par le Sanhédrin (haute cour de justice ${ }^{61}$ ) au sein duquel siège le

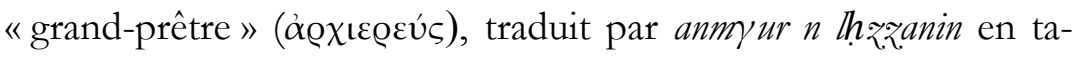
chelhit. Le mot anm $\gamma$ ur, au pluriel inm $\gamma$ urn (de la racine $m \gamma r / m q r$, « être grand ${ }^{62}$ ), «notable, personnage influent », est formé à partir de am (préfixe du nom d'agent) $+m \gamma u r$, avec dissimilation du $m$ en $n^{63}$. Il est suivi de $\operatorname{lh}(a)$ zZan ${ }^{64}$ (au pluriel $\ln (a)$ zzanin), de l'arabe marocain حز حز" rabbin, lettré juif, grand rabbin [mot vague dans l'esprit des musulmans] ; les juifs emploient entre eux le mot rabbi $»^{65}$. On pourrait donc traduire le singulier anmyur n thzzanin par « le grand rabbin » et le pluriel inmyurn n thrzanin par « les principaux rabbins » (littéralement : « les notables des rabbins »).

\footnotetext{
${ }^{59}$ Le numéral $\sin$ (pan-berbère) est proche de l'akkadien šina et de l'hébreu שנים

${ }^{60}$ Littéralement : « le disciple de celui-là ».

${ }^{61}$ Pour la traduction de «Sanhédrin » en tachelhit, voir exemple Jn 11,47.

62 Racine que l'on retrouve dans certains théonymes lybiques et puniques, cf. E. LIPÍNSKI, "Makéris », dans Dieux et déesses de l'univers phénicien et punique, Peeters, (Orientalia Lovaniensia Analecta, 64/Studia Phoenicia 14), Paris-Louvain, 1995, p. 366-369.

${ }^{63}$ De la même racine, est formé le terme amyar, «le chef de la tribu ou du village », voir E. DESTAING, ibid. p. 61.

${ }^{64}$ Le terme apparaît seul en Jn 1,19.

65 A.-L. DE PRÉMARE, ibid., vol. 3, 1994, p. 100.
} 


\section{$\cdot$ Jn 11,47}

Nkrn inmyurn $n$ lhz:anin ${ }^{66}$ d lfarrisiyyin, skern ajmue, ar ttinin : Mamnk ran nsker, ašku argaz ad ar iskar lburban iggutn? (= Alors, les principaux rabbins et les pharisiens convoquèrent ${ }^{67}$ une assemblée (et) dirent: Comment ferons-nous, car cet homme accomplit beaucoup de miracles?)

Le verbe nkr, "se lever», a une valeur inchoative, "se mettre à ", mais il peut aussi exprimer une ponctuation de récit (il correspond dans ce cas à oũv). Pour

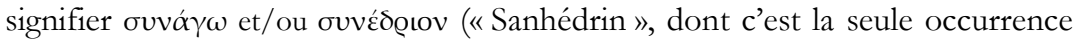
dans le texte johannique) le texte tachelhit emploie le mot ajmuc, «assemblée de notables, d'une tribu ${ }^{68}$, conformément à l'organisation sociale berbère. L'adverbe interrogatif "comment», mamnk est aussi attesté sous la forme manik en tachelhit. On attendrait un $a$ avant la particule modale ran (<rad) cf. manik a ra tskert ("comment feras-tu ?») ${ }^{69}$. Le terme lburhan de l'arabe برهان

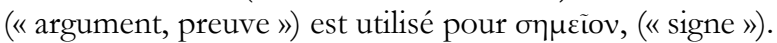

\section{$\cdot$ Jn 18,15 :}

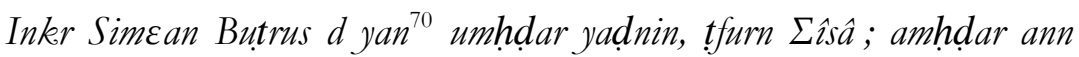
ittyawssan $\gamma$ dar unmyur $n$ Thzzanin ${ }^{71} \ldots$ (= Alors, Simon Pierre et un autre disciple suivirent Jésus ; ce disciple était connu du grand rabbin...)

Le verbe ittyanssan est la forme dérivée passive (composée du préfixe caractéristique $\left.t^{72}\right) 3 \mathrm{~m}$. s. de la $\sqrt{ } \operatorname{snn}$ (« savoir, connaittre $\left.»\right)$.

L'emploi du terme lh(a)zzan montre que les traducteurs ont non seulement tenu compte de la composante judaïque de la société marocaine ${ }^{73}$, mais encore opéré, sur le plan sémantique en tachelhit, une distinction entre les juifs et les chrétiens de l'Evangile de Jean. Ces derniers sont en effet désignés par le biais d'une terminologie islamique susceptible de favoriser l'identification des musulmans à la communauté johannique (cf. lfqib). Toutefois, on ne peut nullement inférer l'existence systématique d'une telle distinction sur la seule base de l'emploi du vocabulaire islamique, comme le montre l'exemple suivant.

${ }^{66} n$ Ihrzanin < llh zzanin (après assimilation progressive totale de $n$ en $\downarrow$ ).

${ }^{67}$ Littéralement : « firent ».

${ }^{68}$ E. Destaing, ibid., p. 23. Cf. l'arabe جمّع "tenir conciliabule », A.-L. DE PRÉMARE, ibid., vol. 2, 1993, p. 226.

${ }^{69}$ E. DesTAING, ibid., p. 71.

70 yan umhdar < ya umhḍar, « un disciple » (après assimilation du $n$ en $u$ ).

71 thrzanin < lh rqaanin (après assimilation du $n$ en $l$ ).

${ }^{72}$ Le préfixe de la forme dérivée passive en tachelhit connait des variantes dont tty et tuw, cf. A. EL MOUNTASSIR, Dictionnaire des verbes Tachelhit-Français (parler berbère du sud du Maroc), L'Harmattan (Tira - Langues, littératures et civilisation berbères), Paris, p. 13. Dans le texte, ce préfixe se présente sous la forme ttyaw.

${ }^{73}$ Les populations juives berbères sont présentes au Maroc (surtout en milieu rural), jusqu'au $20^{\mathrm{e}}$ siècle, cf. H. ZAFRANI, Etudes et recherches sur la vie intellectuelle juive au Maroc: de la fin du $15^{e}$ au début du $20^{\circ}$ siècle. Littératures dialectales et populaires juives en Occident musulman : l'écrit et l'oral, vol. 3, Paris, Geuthner, 2003 (2e édition), p. 21-23. 


\section{- Synagogue}

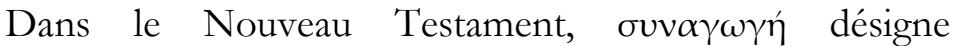
l'«assemblée» ou la «synagogue» (considérée comme lieu d'instruction et d'enseignement ${ }^{74}$. Cité deux fois dans l'Evangile de Jean (Jn 6,59 et 18,20) ${ }^{75}$, le terme est rendu en tachelhit par $t$ (i)magida (au pluriel t(i)magadiwin), "mosquée », forme berbérisée dérivée de l'arabe 76 . La mosquée assure la fonction de lieu de culte mais également de lieu d'apprentissage coranique et religieux $^{77}$, ce qui correspond bien à l'activité de Jésus du point de vue islamo-berbère. Il n'est toutefois pas exclu que l'emploi du mot $t(i) m$ zgida résulte de l'adaptation du lexique judaïque dans un environnement islamique ${ }^{78}$. Cet usage est en effet attesté tant du point de vue juif que du point de vue musulman ${ }^{79}$, aussi bien en arabe marocain qu' «en pays berbère $»^{80}$. L'emploi du terme t(i)magida, qui dépend avant tout de la projection du lecteur, offre par conséquent une double résonnance (d'abord, après le discours de Jésus sur le pain de vie):

\section{$\cdot \mathrm{Jn}, 6,59$ :}

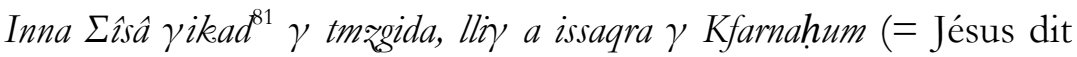
ces choses dans la synagogue/mosquée (des juifs) où il enseignait à Capharnaüm).

La forme verbale issaqra à l'aoriste intensif (< itsaqra, après assimilation du $t$ en $s$ ) est composée de la racine $\gamma r$ («appeler, lire ») ${ }^{82}$ précédée de $s$,

${ }^{74}$ F.G. Vigouroux, «Synagogue », dans Dictionnaire de la Bible, vol. 5/3, Letouzey et Ané, Paris, 1912, col. 1899-1900.

${ }^{75}$ Le mot est employé sans article dans le texte grec. On ne discutera pas ici le caractère philologique de la question, l'objet de ce commentaire portant en priorité sur le texte cible.

${ }^{76}$ N. VAN DEN BOOGERT et M. KOSSMANN, ibid., p. 319.

${ }_{77}$ Destaing lui donne également le sens d' «école », cf. E. DestaInG, ibid., p. 102.

${ }^{78}$ Les juifs berbères parlaient la langue de leur milieu et on connaît des cas où des expressions musulmanes sont passées dans certains parlers juifs marocains, cf. M. BAR ASHER, «Vestiges islamiques dans le parler judéo-arabe du Maroc », dans Journal Asiatique, 292, 1-2, 2004, p. 361-380.

${ }^{79}$ Dans ce cas, il est généralement suivi de lyabūd (= «la mosquée des juifs »), de même qu'on a « la mosquée des chrétiens » pour désigner l'église, cf. P. WEXLER, «Terms for 'synagogue' in hebrew and jewish languages. Explorations in historical jewish interlinguistics ", dans Jewish and Non-Jewish Creators of "Jewish" Languages, Harrassowitz, Wiesbaden, 2006, p. 131-132. Voir aussi A.-L. DE PRÉMARE, ibid., vol. 2,1993, p. 229.

${ }^{80} \mathrm{H}$. ZAFRANI, Mille ans de vie juive au Maroc : histoire et culture, religion et magie, Maisonneuve \& Larose, Paris, 1983, vol.1, p. 62.

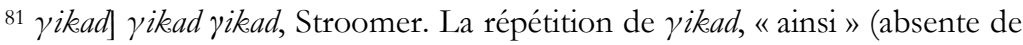
l'édition princeps) n'est pas fausse. Littéralement «ainsi, ainsi» a pour sens « ceci et cela» ou «telle chose». C'est l'équivalent de l'arabe marocain كذا كذا $(<k a+d h \vec{a})$ «comme ceci, comme cela »), cf. A.-L. DE PRÉMARE, ibid., vol. 10, 1998, p. 535. La répétition figure également dans la transcription de Roux. C'est peut-être une leçon propre à la révision de 1925. 
marque de la forme causative commune au groupe afro-asiatique ${ }^{83}=$ " enseigner, instruire » (littéralement « faire lire »).

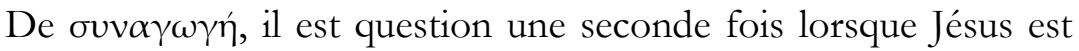
interrogé par le grand prêtre sur la nature de son enseignement :

\section{$\cdot$ Jn 18,20 :}

... ar bdda ssaqray $\gamma$ tmagadiwin ula $\gamma$ Bit Lquds $\gamma i$ lli $\gamma$ bdda ttmunn wudayn, ur jju sntihy awal inu. (= ... j'ai toujours enseigné dans les synagogues/mosquées (des juifs) ainsi que dans le Temple où les juifs ${ }^{84}$ se réunissent toujours (et) je n’ai jamais caché mon propos).

Le grec donne $\dot{\varepsilon} v \sigma u v \alpha \gamma \omega \gamma \tilde{\eta}$ au singulier tandis que le tachelhit utilise

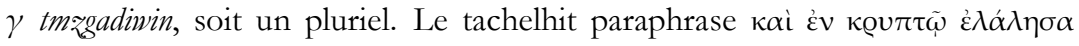
oủঠév (" et je n'ai rien dit en secret») en "je n'ai jamais caché mon propos». L'adverbe «toujours", bdda, dérive de l'arabe أبدا. Le verbe mun, « être avec, aller avec, accompagner, se réunir $»^{85}$ est à l'aoriste intensif (marque l'habitude). Le Temple (íøóv), centre du culte mosaïque, est rendu par Bit Lquds, emprunt morphologique et lexical arabe de Bit (القسب, " Jérusa-

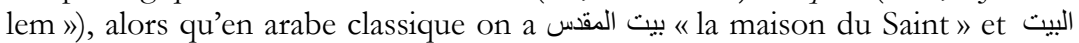
"la maison Sainte » ${ }^{86}$. Dans la traduction, "Jérusalem» est rendu par Imdint $n$ bit lquds (= "la ville de la maison/temple de Jérusalem »). Toutefois, lorsque Jésus parle du «Temple » de son corps (en Jn 2,19-20 et 21), le mot est traduit par lbit n Rbbi, "la maison de Dieu », allusion explicite au mystère de l'Incarnation.

Ce dernier exemple appelle une remarque. On peut en effet comprendre le passage comme suit : "J'ai toujours enseigné dans les synagogues (=les mosquées des juifs) et dans le Temple où les juifs se réunissent...», ou encore: "J'ai toujours enseigné dans les mosquées et dans le Temple où les juifs se réunissent...». Dans ce dernier cas, le verset prend une dimension exégétique précise et peut être interprété comme un signe de l'universalité de la mission de Jésus, du point de vue tant islamique que chrétien.

${ }^{82}$ La racine qra est commune au sémitique (phénicien, hébreu, araméen, syriaque, arabe, nabatéen et palmyrénien). Ici, la vélaire sourde $q$ est passé à la vélaire sonore $\gamma$ et réapparaît à certaines formes de la conjugaison du verbe.

${ }^{83} \mathrm{~J}$.-C. HAELEWYCK, ibid., p. 134.

84 " Juifs ", udayn (au singulier uday), dériverait du latin iudaens, cf. V. BRUGNATELLI, "Uday 'ebreo' e Israel in North Africa », dans C. RosENZWEIG, A.L. Callow, V. Brugnatelli, F. Aspesi (éds.), Florilegio filologico linguistico. Haninura de Bon Siman a Maria Luisa Mayer Modena, Cisalpino, Milano, 2008, p. 47-54.

85 A. El MOUNTASSIR, ibid., p. 127.

${ }^{86}$ A.B. KASIMIRSKI, ibid., vol. 1, p. 181-182. 


\section{Remarques générales}

De ces observations d'ensemble, il ressort que l'Evangile de Jean en tachelhit a été adapté au substrat de la religion dominante du milieu de réception. Il est toutefois peu probable que cette « contextualisation » soit le fruit de l'adaptation de l'Evangile de Jean au substrat judaïque formulé en terre d'islam. Il semble en effet que les traducteurs aient procédé à dessein à des choix lexicaux permettant l'identification du public musulman, principal destinataire, au noyau de la communauté de Jésus, Jean-Baptiste et des disciples.

En outre, on a vu que l'emploi du lexique religieux berbère tachelhit faisait état d'emprunts à la langue arabe, difficilement dissociable de son substrat islamique au Maghreb. Il s'ensuit une relative continuité entre la terminologie musulmane et le vocabulaire biblique.

Ce modèle d'adaptation de l'Evangile pourrait certes faire l'objet de débats sur le plan missiologique. En ce sens, la traduction de l'Evangile de Jean en berbère tachelhit constitue un document digne d'intérêt : il présente en effet un témoignage indirect de la pratique missionnaire dans le Sud marocain au début du $20^{\mathrm{e}}$ siècle, mais il offre aussi, plus largement, un écho de présence chrétienne en Afrique du Nord. 
Reproduction

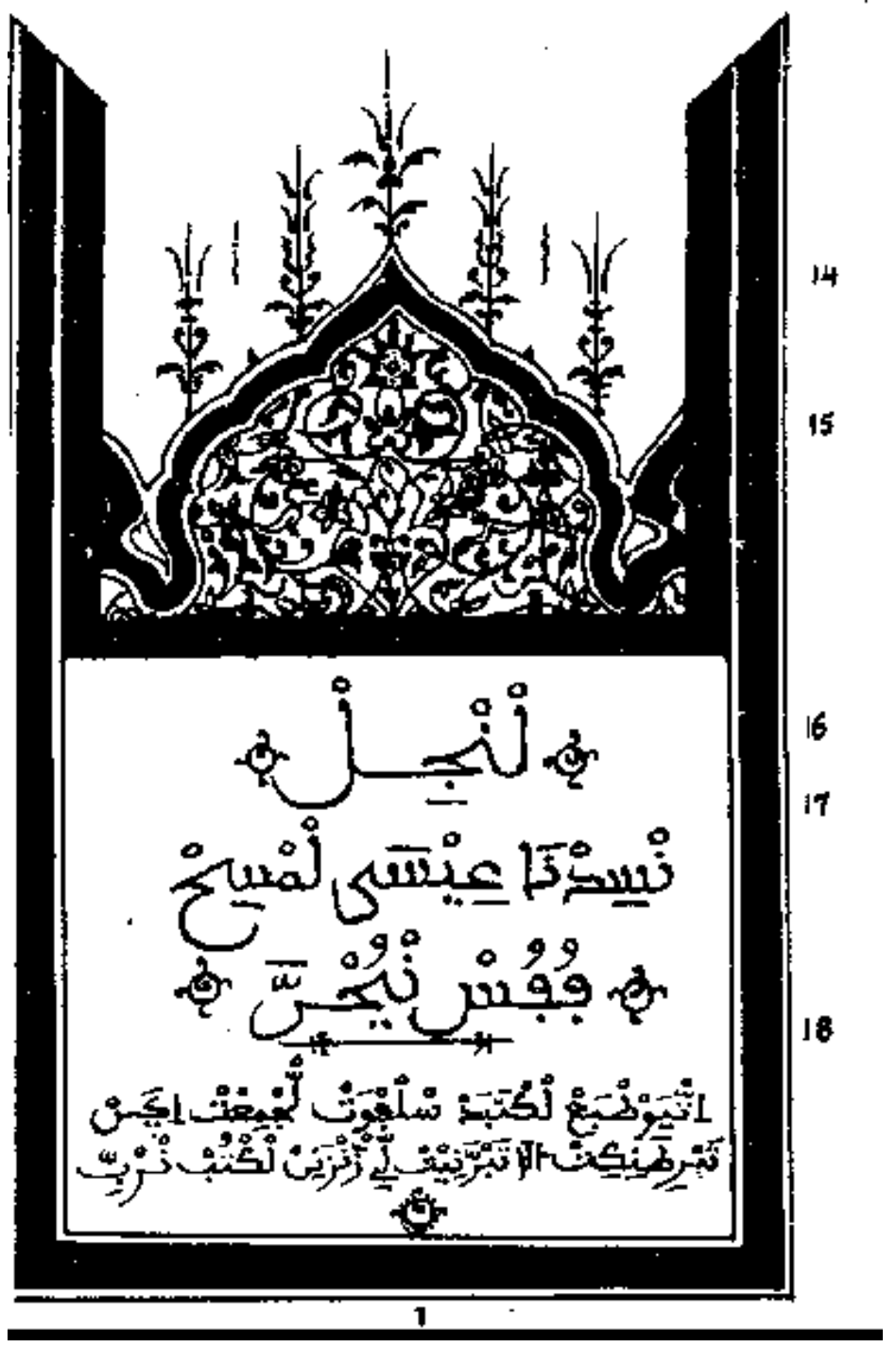

Page de garde de la traduction de l'Evangile selon Saint Jean en berbère tachelhit publiée par la British and Foreign Bible Society (1906). Après le titre (souligné), les deux lignes mentionnent: ittyawtbae lktab ad s lfwat n limict igan tabritanikt ula tabrraniyt lli zznzanin lktub n Rbbi (= «Ce livre a été imprimé avec le soutien financier de la Société britannique et étrangère chargée de la vente des Saintes Ecritures [litt. livres de Dieu] »). 